\title{
Accuracy of Ultrasound Scanning in Diagnosing Acute Appendicitis in Patients Admitted at Vicente Sotto Memorial Medical Center,
}

\section{*Uma Gurung, ${ }^{1}$ Dhiraj Gurung ${ }^{2}$}

${ }^{1}$ Lecturer, Department of Radiology, ${ }^{2}$ Assistant Professor, Department of Internal Medicine; Karnali Academy of health sciences, Jumla, Nepal of Internal Medicine; Karnali Academy of health sciences, Jumla, Nepal

*Corresponding Author: Dr Uma Gurung; Email- umagrg1128@gmail.com

\begin{abstract}
Introduction: Acute appendicitis is the most common abdominal surgical emergency. Both abdominal ultrasonography and computed tomography are common diagnostic tools in its diagnosis with each having its own advantages and disadvantages.

Methods: Patients of suspected acute appendicitis were evaluated with an ultrasound to see the sensitivity, specificity, positive and negative predictive value of ultrasound for intraoperative appendicitis diagnosis. The study included 113 patients of suspected acute appendicitis presenting in the emergency during a one year duration. Sensitivity, specificity, positive predictive value and negative predictive value was calculated from their respective formulae.

Results: The majority of the patients were male patients between the age group of 18 to 30 . The sensitivity of ultrasound for diagnosis of acute appendicitis was $96 \%$ and specificity was $33 \%$. The positive predictive value was $98 \%$ and the negative predictive value was $20 \%$

Conclusion: Ultrasound has good sensitivity and the low cost along with no radiation exposure makes this an acceptable screening investigative modality though due to low specificity, it would be recommended to go for a computed tomography scan if ultrasound shows negative result for appendicitis.
\end{abstract}

Keywords: ultrasound, computed tomography, appendicitis

\begin{tabular}{|c|c|c|}
\hline \multicolumn{2}{|c|}{ Access this article Online } & Article Info. \\
\hline Quick Response Code & Website: & How to cite this article in Vancouver Style? \\
\hline & www.jkahs.org.np & $\begin{array}{l}\text { Gurung U, Gurung D. Accuracy of ultrasound } \\
\text { scanning in diagnosing acute appendicitis in patients } \\
\text { admitted at Vicente Sotto Memorial Medical Center. } \\
\text { Journal of Karnali Academy of Health Sciences } \\
\text { 2020;3(2): 134-139 }\end{array}$ \\
\hline & $\begin{array}{l}\text { DOI: } \\
\text { https://doi.org/10.3126/ } \\
\text { jkahs.v3i2.31325 }\end{array}$ & $\begin{array}{ll}\text { Received } & : \text { 12 June2020 } \\
\text { Accepted } & : 9 \text { August } 2020 \\
\text { Published Online } & : \text { 10 August } 2020 \\
& \\
\text { Conflict of Interest } & \text { : None } \\
\text { Source of Support } & : \text { None }\end{array}$ \\
\hline
\end{tabular}




\section{INTRODUCTION}

Acute appendicitis is the most common abdominal surgical emergency. ${ }^{1}$ The typical clinical presentation of appendicitis is initial periumbilical abdominal pain which is progressively localized to a point in the right lower quadrant (RLQ) where the inflamed appendix irritates the parietal peritoneum. The pain is usually associated with fever, vomiting and leukocytosis. ${ }^{2}$ However patients with atypical presentations can cause diagnostic problems. The most difficult patients are women of child bearing age in whom ruptured ovarian cysts and pelvic inflammatory disease may mimic acute appendicitis. ${ }^{3}$

Imaging for appendicitis allows for confirmation of the diagnosis prior to an invasive procedure. Plain $\mathrm{x}$-ray-film show a very low diagnostic value for acute appendicitis. ${ }^{4}$ The two most common investigation used for diagnosis of acute appendicitis are abdominal ultrasound and abdominal computed tomography. ${ }^{5}$

According to the ACR, computed tomography is the most accurate imaging study for evaluating suspected acute appendicitis. It is especially useful in those suspected of appendiceal perforation and is useful for characterizing peri-appendiceal inflammatory masses. Computed Tomography scanning is the gold standard for the diagnosis of appendicitis but it suffers from drawbacks like exposure to ionizing radiation. ${ }^{6}$

Various proposed protocols have kept ultrasound as the first diagnostic modality with Computed Tomography scan being used only if the ultrasound is negative or equivocal for acute appendicitis. ${ }^{7}$, ${ }^{8,} 9$ Graded-compression Ultrasound use in acute appendicitis has now increased dramatically. ${ }^{10,11}$ Meta-analysis and other studies show the sensitivity of ultrasound as $88 \%$ and specificity as $94 \%$ for children while for adults, the sensitivity is $83 \%$ and its specificity $93 \%{ }^{12-16}$

This study would add on to the already accepted practice of using and training for ultrasound use in suspected acute appendicitis. In pregnant women, ultrasound is the preferred diagnostic modality with MRI being used in inconclusive cases. ${ }^{17}$
The American College of Radiology (ACR) have published appropriateness criteria for right lower quadrant pain suggestive of appendicitis. In this criteria, ratings of 7 to 9 are considered "usually appropriate." Ultrasonography of the right lower quadrant with graded compression is rated $6 .{ }^{18}$ It has been shown that even non-radiologists can obtain specificities as high as radiologists with ultrasound. ${ }^{19}$

Retrocecal position of the appendix results in missed diagnosis of acute appendicitis in about $30 \%$ of the cases. This can be partially overcome by scanning in the coronal plane to visualize posterior to the cecum..$^{20}$ The objective of this study is to see the sensitivity and specificity of ultrasonography for use in the diagnosis of acute appendicitis.

\section{MARTIALS AND METHODS}

This was a retrospective cross-sectional diagnostic validation study conducted in Vicente Sotto Memorial Medical Center, Cebu city. A total of 113 patients with age range of 18-99 years old who present with suspected diagnosis of acute appendicitis and underwent ultrasonography prior to treatment in the ER from Feb 2015 to Feb 2016.

Data was collected to include name, age, sex, ultrasound diagnosis, intraoperative diagnosis.

Ethical approval for the study was obtained from the local research council of the centre.

Appendicitis was defined here as intraoperative finding of inflammation of the appendix resulting from obstruction of its lumen from stones, lymph node enlargement, etc.

Acute appendicitis was suspected on symptons of right lower or periumbilical abdominal pain, fever and/or leukocytosis. Inclusion criteria was all patients admitted at Vicente Sotto Memorial Medical Center, Cebu city with age range of 18-99yrs old who presents with right lower or periumbilical abdominal pain, fever and/or leukocytosis at ER from Feb 2016 who are suspected of having acute appendicitis and undergo ultrasound before treatment is established while those with incomplete records and those who have already undergone appendectomy at the time of presentation was excluded. 
Sensitivity, specificity, positive and negative predictive values of ultrasound for the diagnosis of acute appendicitis will be calculated using the following formula-

\begin{tabular}{|l|l|l|}
\multicolumn{1}{|c|}{ Test } & Disease present & Disease absent \\
\hline Positive & A & B \\
\hline negative & C & D \\
\hline
\end{tabular}

Sensitivity- $a / a+c$

Specificity- $d / b+d$

Positive predictive value- $\mathrm{a} / \mathrm{a}+\mathrm{b}$

Negative predictive value- $d / c+d$

\section{Study limitations}

Use of ultrasound can result in the over-diagnosis of appendicitis. We can sometimes misinterpret the terminal ileum as the appendix while sometimes a normal appendix has been taken for an inflamed appendix. A gas-filled appendix can be misinterpreted as a small-bowel loop.

Factors such as obesity, severe pain or abdominal guarding, excessive bowel gas and an uncooperative patient can all affect the accuracy of the ultrasound due to the sonographer being unable to achieve adequate compression of the study site. Operator experience can also affect the study result.

Histo-pathological findings, which would be the gold standard for diagnosing appendicitis, has not been included here due to it being unavailable for some of our patients. Instead the diagnosis of appendicitis was made by intraoperative notes regarding finding of inflamed edematous sometimes suppurative perforated appendix.

\section{RESULTS}

Total of 113 patients met the inclusion criteria for the study. The age distribution of the patients showed predominance of young patients below 50 years of age. There were a total of 78 male patients versus 35 female patients.

The study showed a sensitivity of $96 \%$, Specificity of $33 \%$, Positive Predictive Value of $98 \%$ and a Negative Predictive Value of $20 \%$.

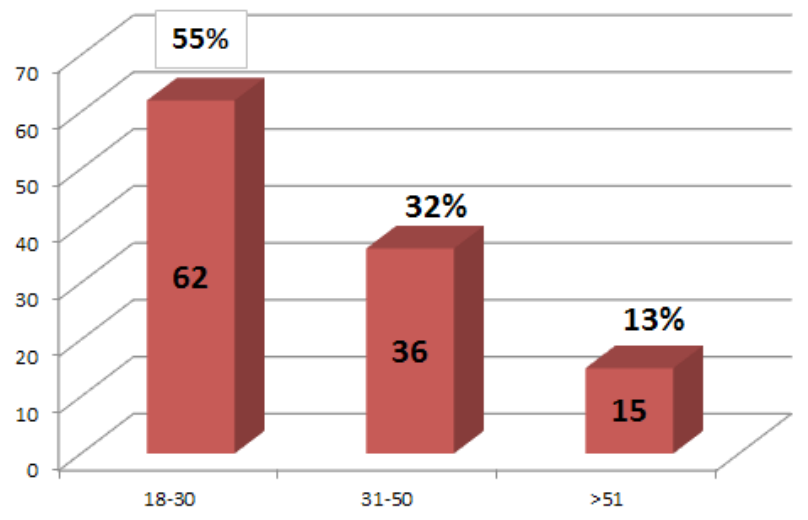

Figure 1. Age distribution- total 113 patients with 62 patients between ages of 18 to 30,36 between ages 31 to 50 and 15 greater than 51 years of age. The oldest patient was 72 years of age while the youngest was 18 years of age

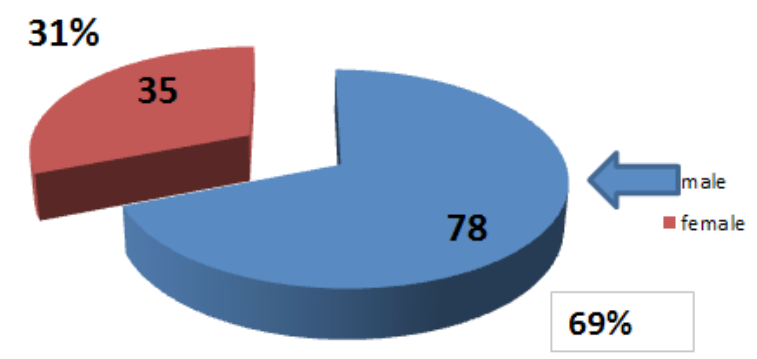

Figure 2. Sex distribution- this study had a total of 78 male patients versus 35 female patients

Table 1. Data analysis- chart showing true positive of $\mathbf{1 0 6}$ and true negative of $\mathbf{1}$

\begin{tabular}{|l|c|c|c|}
\hline \multicolumn{2}{|c|}{ Appendicitis by intraoperative finding (+ve) } & $\begin{array}{c}\text { Not appendicitis by intraoperative } \\
\text { finding (-ve) }\end{array}$ & Total \\
\hline Appendicitis by USG (+ve) & 106 & 2 & 108 \\
\hline Not appendicitis by USG (-ve) & 4 & 1 & 5 \\
\hline Total & 110 & 3 & 113 \\
\hline
\end{tabular}

Sensitivity $=106 / 110 \times 100=96 \%$

Specificity $=1 / 3 \times 100=33 \%$

Positive Predictive Value $=106 / 108 \times 100=98 \%$

Negative Predictive Value $=1 / 5 \mathrm{X} 100=20 \%$ 


\section{DISCUSSION}

Acute appendicitis is the most common abdominal surgical emergency. ${ }^{1}$ The typical clinical presentation of appendicitis may not always be present and can cause diagnostic problems while other conditions mimic appendicitis like ruptured ovarian cysts and pelvic inflammatory disease. ${ }^{3}$ Diagnostic difficulties such as these highlight the role of imaging studies with abdominal ultrasound and abdominal computed tomography. ${ }^{4}$ Various studies have shown ultrasound to have comparable sensitivity and specificity compared to computed tomography besides other advantages like radiation and cost. ${ }^{21}$

This study has shown a wide age distribution among the patients with the oldest patient being 72 years old. Patients younger than 18 years have been excluded to simplify the data collection process hence the difference from other studies where children as young as 3 years of age were diagnosed with appendicitis. ${ }^{6}$ This study also shows a higher male to female ratio which is similar to findings in other studies of similar population as well as amongst the western population. ${ }^{14}$

This study has shown ultrasound of having high sensitivity (96\%) and positive predictive value (98\%) which is comparable to other similar studies. ${ }^{4}$

This high sensitivity profile is comparable to that of contrast enhanced computed tomography (98\%) and better than non-contrast computed tomography $(66 \%){ }^{10}$ Acute appendicitis is a diagnosis where one would rather open up the patient and be wrong than wait and watch and risk perforation and peritonitis. The high sensitivity of ultrasound makes it a good investigation to rule in appendicitis which is the basis of various protocols that use ultrasound as the first investigative modality with follow up computed tomography in cases of equivocal or negative results.

This study shows a specificity of $33 \%$ which is lower compared to other similar studies with some studies showing specificity as high as $94 \%$. This could be due to the following reasons-

- Limited experience of the resident
ultrasonologist,
- Inadequate use of intravenous short acting analgesics during ultrasound in this facility. The inability to tolerate compression by the ultrasound probe may obscure the image, decreasing the accuracy of the scan due to bowel gas especially as these patients have not undergone any bowel preparation.

An experienced ultrasonologist could have better results in terms of specificity so a second look by a consultant ultrasonologist can be a recommended step if equivocal or negative results are obtained.

Multi-detector Computed tomography has been shown to have a high specificity of up to $98 \%$. One could argue that ideally a test with both high sensitivity and specificity like contrast enhanced computed tomography should be employed as the diagnostic modality of choice versus ultrasonography. It would diagnose as many cases as possible while at the same time limit the number of false positive resulting in decrease in unnecessary laparotomy. This reasoning does not take into consideration the cost of the investigation, the adverse effects of the investigation and contrast. The patient must be able to firstly afford the investigation before he or she is able to do it. The radiation from computed tomography limits its use in pregnant patients and children. The use of contrast agents is associated with its own risk of adverse events important amongst them being anaphylaxis. For these particular subset of patients, ultrasound can definitely be taken as the first investigative modality of choice. This study is comparable to other studies where they have compared ultrasound diagnosis of appendicitis to intraoperative finding of acute appendicitis. $^{22}$

\section{CONCLUSION}

Ultrasound has good sensitivity and the low cost along with no radiation exposure makes this an acceptable screening investigative modality though due to low specificity, it would be recommended to go for a computed tomography scan if ultrasound shows negative result for appendicitis It can be used as a first line investigative modality for children, pregnant women and the financially weak patients. 


\section{REFERENCES}

1. Gupta R. Textbook of surgery, $2^{\text {nd }}$ Ed. Jaypee brothers medical publishers; 2003. 49: 939

2. Wijetunga R, Tan BS, Rouse JS, Bigg-Wither GW, Doust BD. Diagnostic accuracy of focused appendiceal CT in clinically equivocal cases of acute appendicitis. Radiol. 2001. 221:74753. https://doi.org/10.1148/radiol.2213001581 [Pubmed] [Google Scholar]

3. Brant WE, Helms CA. Fundamentals of diagnostic radiology, $3^{\text {rd }}$ Ed. Lippincott Williams and wilkins; 2007. 32: 861

4. Rao PM, Rhea JT, Rao JA, Conn AK. Plain abdominal radiography in clinically suspected appendicitis: diagnostic yield, resource use, and comparison with CT. Am J Emerg Med. 1999 Jul. 17(4): 325-8. https:// doi.org/10.1016/ $\underline{\text { s0735-6757(99)90077-3 [Pubmed] [Google }}$ Scholar]

5. Reich B, Zault T, Weiner SG. An international evaluation of ultrasound vs. computed tomography in the diagnosis of appendicitis. Int J EmergMed. 2011. 4: 68. https://doi. org/10.1186/1865-1380-4-68

[Pubmed] [Google Scholar]

6. Brenner DJ, Hall EJ. Computed tomography- an increasing source of radiation exposure. NEJM. 2007;35(22):2277-84. https://doi: 10.1056/ NEJMra072149. [Pubmed] [Google Scholar]

7. Poortman P, Oostvogel HJ, Bosma E, Lohle $\underline{\text { PN, Cuesta MA }}$ Hamming JF, et al. Improving diagnosis of acute appendicitis: results of a diagnostic pathway with standard use of ultrasonography followed by selective use of CT. J Am Coll Surg. 2009 Mar. 208(3):434-41. http://doi: 10.1016/j.jamcollsurg.2008.12.003 [Pubmed] [Google Scholar]

8. Garcia PBM, Mandl KD, Kraus SJ, Fischer AC,Fleisher GR, Lund DP, etal. Ultrasonography and limited computed tomography in the diagnosis and management of appendicitis in children. JAMA 199915;282:1014-6. . . http:// doi:10.1001/jama.282.11.1041 [Pubmed] [Google Scholar]

9. Ramarajan N, Krishnamoorthi R, Barth R, Ghanouni P, Mueller C, Dannenburg B, et al. An interdisciplinary initiative to reduce radiation exposure: Evaluation of appendicitis in a pediatric emergency department with clinical assessment supported by a staged ultrasound and computed tomography pathway. Acad Emerg Med. 2009;16(11): 1258-65. http:// doi:10.1111/j.1553-2712.2009.00511.x.

\section{[Google Scholar]}

10. Puylaert JB. Acute appendicitis: US evaluation using graded compression. Radiol. 1986 Feb. 158(2):355-60. $\quad$ http://doi:10.1148/ radiology.158.2.2934762 [Google Scholar] [Pubmed]

11. van Randen $\mathrm{A}$, Bipat $\mathrm{S}$, Zwinderman $\mathrm{AH}$, Ubbink DT, Stoker J, Boermeester MA. Acute appendicitis: meta-analysis of diagnostic performance of CT and graded compression US related to prevalence of disease.Radiol. 2008 Oct. 249(1):97-106. http://doi: 10.1148/ radiol.2483071652.

\section{[Google Scholar] [ $\underline{\text { Pubmed] }}$}

12. Doria AS, Moineddin R, Kellenberger CJ,Epelman M, Beyene J, Schuh S,et al. US or CT for the diagnosis of appendicitis in children and adults? A meta-analysis. Radiol 2006;241(1): 83-94. http://doi: 10.1148/ radiol.2411050913.

\section{[Google Scholar] [Pubmed]}

13. Quillin SP, Siegel MJ. Appendicitis: efficacy of color Doppler sonography. Radiol. 1994 May. 191(2):557-60. http://doi: 10.1148/ radiology.191.2.8153340.

[Google Scholar] [Pubmed] 
14. Patriquin HB, Garcier JM, Lafortune M, Yazbeck $\mathrm{S}$, Russo P, Jequier S, et al. Appendicitis in children and young adults: Doppler sonographicpathologic correlation. AJR Am J Roentgenol. 1996 Mar. 166(3): 629-33. http://doi: 10.2214/ ajr.166.3.8623640.

\section{[Google Scholar] [Pubmed]}

15. Brown MA. Imaging acute appendicitis. Semin. Ultrasound CT MR. 2008;29:293-307. http:// doi: 10.1053/j.sult.2008.06.003

\section{[Google Scholar] [Pubmed]}

16. Lee JH, Jeong YK, Hwang JC, Ham SY, Yang SO. et al. Graded compression sonography with adjuvant use of a posterior manual compression technique in the sonographic diagnosis of acute appendicitis. Am J Roentgenol. 2002;178(4):863-8. http://doi: 10.2214/ ajr.178.4.1780863.

\section{[Google Scholar] [Pubmed]}

17. Smith MP, Katz DS, Lalani T, Carucci LR, Cash BD, Kim DH, et al. ACR Appropriateness Criteria ${ }^{\circledR}$ Right Lower Quadrant Pain-Suspected Appendicitis. Ultrasound $Q$. 2015 Jun. 31 (2):85-91. http://doi: 10.1097/ RUQ.0000000000000118 [Pubmed]

18. Rosen MP, Ding A, Blake MA, Baker ME, Cash BD, Fidler JL, et al. ACR Appropriateness Criteria ${ }^{\circledR}$ right lower quadrant pain-suspected appendicitis. J Am Coll Radiol. 2011 Nov. 8(11):749-55. http://doi: 10.1016/j. jacr.2011.07.010.

[Google Scholar] [ubmed]
19. Pohl D, Golub R, Schwartz GE, Stein HD. Appendiceal ultrasonograpy performed by non-radiologist: Does it help in the diagnostic process? J. Ultrasound Med. 1998;17(4) :21721.[Google Scholar] [Pubmed]

20. Krishnamoorthi R, Ramarajan N, Wang NE, Newman B, Rubesova E, Mueller CM, et al. Effectiveness of a staged US and CT protocol for the diagnosis of pediatric appendicitis: reducing radiation exposure in the age of ALARA. Radiol. 2011 Apr. 259(1): 231-9. https://doi.org/10.1148/radiol.10100984

[Google Scholar] [Pubmed]

21. Bakker OJ, Go PM, Puylaert JB, Kazemier G, Heij HA. Werkgroep richtlijn Diagnostiek en behandeling van acute appendicitis. Ned Tijdschr Geneeskd. 2010;154:A303

[Full Text]

22. Laxman S, Sangolgi P. Accuracy of ultrasonography in the diagnosis of acute appendicitis. IJSS journal of surgery 2018; 4(1):1-4. [Google Scholar] 